\title{
Extracorporeal Shock Wave Versus Interferential Current in Tennis Elbow
}

\author{
Tarek Ammar* \\ Professor, Faculty of Physical Therapy, Cairo University, Cairo, Egypt \\ *Corresponding author: Tarek Ammar, Professor, Faculty of Physical Therapy, Cairo University, Cairo, Egypt
}

ARTICLE INFO

Received: 慧 March 01, 2021

Published: 幽 March 11, 2021

Citation: Tarek Ammar. Extracorporeal Shock Wave Versus Interferential Current in Tennis Elbow. Biomed J Sci \& Tech Res 34(3)-2021. BJSTR. MS.ID.005561.

Keywords: Tennis Elbow; Shock Waves; Interferential Current; Pain
ABSTRACT

Objective: The purpose of this study was to compare between the effects of Extracorporeal Shock Wave Therapy (ESWT) and Interferential Therapy (IFC) in improving pain intensity, disability and grip strength in patients with Tennis Elbow (TE).

Setting: A physical therapy outpatient clinic.

Participants: Seventy-two participants with TE completed the program and were randomly assigned into three groups. Pain Duration was more than 12 weeks.

Interventions: Group $1(\mathrm{n}=24$, mean age $=40.41 \pm 3.91$ years $)$ received ESWT and exercises. Group2 ( $\mathrm{n}=24$, mean age $=44.72 \pm 7.10$ years) received IFC and exercises. Group $3(n=24$, mean age $=49.01 \pm 3$.61years $)$ received exercises. All subjects received three visits a week for four weeks.

Materials: Visual analog scale, the disabilities of the arm, shoulder and hand questionnaire and the hand dynamometer were used to measure pain intensity, disability and grip strength before and after 4 weeks of intervention.

Results: There were statistically significant improvements in all dependent variables. Also, subjects who received ESWT and exercises had the most significant effects. ESWT may be more effective than IFC in treating TE by reducing pain intensity, disability and increasing grip strength.

Conclusion: ESWT improved pain, function and disability better than IFC in patients with TE.

\section{Introduction}

Tennis Elbow (TE) encompasses pain and tenderness due to overuse or injury of the extensor wrist tendons [1]. It is a degenerative rather than inflammatory tendinopathy causing persistent elbow pain [2]. Moreover, TE usually affects 4-7 per 1000 individuals [3]. A typical episode lasts 6-24 months and recurrence is common. They also reported that conditions that are not resolved within six months of the onset, may need surgical interventions. Modalities include but are not limited to acupuncture, splinting, therapeutic exercises, shock wave therapy, laser, ultrasound, transcutaneous electrical nerve stimulation, manipulation, and manual therapy [4,5]. Unfortunately, there is no consensus on the optimum intervention strategy for TE. As such, research for new effective interventions for TE is warranted. Extracorporeal Shock
Wave Therapy (ESWT) is used to treat many musculoskeletal disorders such as plantar fasciitis, calcific tendinitis of the shoulder, epicondylitis, patellar tendinopathy, Achilles's tendinopathy, nonunion and delayed union of long bone fracture [6]. ESWT may impede progression of $\mathrm{OA}$, improve function, and depress chondro protective effects in animals $[7,8]$. A few researchers elucidated that ESWT reduced pain and improved knee functions in patients with knee OA $[9,10]$. Therefore, ESWT has been suggested effective for TE. Interferential Current (IFC) has been used for treating musculoskeletal disorders for its analgesic, anti-inflammatory, sympatholytic, local vasodilating, and muscle stimulating effects [11]. IFC is also used to reduce pain in sports injuries, arthritis, low back pain, Osgood-Schlatter disease, rheumatoid arthritis, 
and muscular pain [12]. However, there has been little research comparing between ESWT and IFC in patients with knee OA. Also, little is known about the efficacy of these two therapies in treating TE. The purpose of this study was to determine the effectiveness of ESWT compared with IFC for patients with TE.

\section{Methods}

\section{Design}

This study was a randomized controlled trial with subjects randomly assigned to one of three intervention groups:

a) Group 1 received ESWT and exercises,

b) Group 2 received IFC and exercises, while

c) Group 3 received only exercises.

The examiner made group comparisons at the initial visit and after 4 weeks. The duration of the interventions was 4 weeks per participant. Each participant received one session per week for 4 weeks and 3 sessions per week for four weeks in the second group. The therapist who did the testing and data analyses was not aware of group allocation. However, the treating therapist was aware of group allocation.

\section{Participants}

Eighty-eight participants with TE who visited the outpatient setting for a minimum of 3 months pain duration participated in the study. All participants signed informed consent forms detailing procedure and risks involved with participating in this research. Inclusion criteria included subjects with lateral elbow and forearm pain that lasted for more than six weeks. Subjects had a medical diagnosis of TE by a physician. Subjects experienced less pain during resistance of supination with the elbow in $90^{\circ}$ of flexion rather than in full extension. Patients also had to complain of increased tenderness upon extending the wrist against resistance with the forearm in supination. The exclusion criteria for the study were bilateral TE, systemic metabolic diseases, elbow surgery, medial epicondylopathy, radial nerve entrapment, carpal tunnel syndrome, chronic inflammatory and neoplastic disease; other criteria included: radial tunnel syndrome, cervical radiculopathy, thoracic outlet syndrome, dysfunction in the shoulder, and treatment with corticosteroid or local anesthetic injection in the previous three months. Also, subjects with any contraindication for the physical therapy modalities were excluded. Participants were asked to refrain from receiving other forms of physical therapy and analgesics during the study.

\section{Measurements}

A $10^{-\mathrm{cm}}$ Visual Analog Scale (VAS) was used to measure pain intensity during rest, activity of the painful elbow, and nighttime. It has an excellent test-retest reliability of 0.95 [13]. The disabilities of the arm, shoulder and hand (DASH) self-report questionnaire was also used to measure disability. It is a valid and reliable 30 items questionnaire where 1 point was given for performance of the specific activity without difficulty and 5 points for disability [14]. The total score ranged between 30 (best) and 150 (worst). Handheld dynamometer (Jamar, J.A. Preston Co., MI) was also used as a valid and reliable tool to measure hand grip strength [15]. The assessor used the mean of three measurements. The Shock Master was used to produce shock waves. It is a low to medium-energy range. Evotron RFL0300 (Swiss Tech Medical AG, Switzerland) was used to deliver shock waves. It has a depth of penetration between 0 to $30 \mathrm{~mm}$. Pulse rates were 60,120, 180, 240 impulses per minute. Also, the SONOSTIM (Class 1-type BF, Norm: 601-1) was used to produce IFC.

\section{Interventions}

Patients signed an informed consent before data collection. The research therapist examined all participants to check for inclusion and exclusion criteria and was not aware of the intervention assignments. He tested the participants at both the baseline and final sessions. Another therapist performed all interventions. All subjects received three sessions per week for four weeks. Patients in the first group received a total of four sessions of ESWT. They received one session a week for four weeks. The patient was placed in supine lying position with knees bent to 90o. The therapist applied 1,000 pulses of shockwave with an energy dose of $0.05 \mathrm{~mJ} /$ $\mathrm{mm}^{2}$. He directed it to over the common extensor tendon and the other electrode proximal to the olecranon. Participants in the second group received the IFC. The therapist placed the four electrodes around the elbow so that each channel runs perpendicular to the other and the two current crosses at a midpoint in the center of the elbow.

He adjusted the intensity in the tactile sensation threshold. IFC was conducted with following characteristics: isoplanar vector field with 6:6 sweep mode; carrier frequency $4 \mathrm{kHz}$; beat frequency 100 $\mathrm{Hz}$; and sweep frequency $150 \mathrm{~Hz}$. The duration of the stimulation was 20 minutes. The therapist increased the current amplitude until the participant felt a strong but comfortable tingling. He increased the intensity until the participant reached the previous sensation in case of sensory habituation. Participants in the third group received therapeutic exercises. Also, participants of the first and second group received the same therapeutic exercises. Exercises were progressive eccentric exercises of the wrist extensors and stretching exercises of the extensor carpi radialis brevis for 20 minutes. Eccentric exercises were done by asking the subject to put the elbow in extension, forearm in pronation, the wrist in extension, and the hand hanging over the edge of the bed.

Then, the therapist asked the subject to bend the wrist slowly while counting to 30 and then return to the starting position with the assistance of the other hand. The therapist asked the subjects 
to exercise within their pain tolerance. Stretching exercises were done by asking each subject to maintain the elbow in full extension, forearm in full pronation, and the wrist in flexion and ulnar deviation within pain tolerance for 45 seconds. The therapist asked participants to record frequency and repetition of each exercise to measure adherence to exercises. The therapist asked each subject to avoid activities that may irritate the elbow such as shaking hands, grasping, or lifting. The therapist advised the subjects not to take pain medications one week before the visits. The therapist also instructed the subjects to do the home exercises on the days they do not come to the clinic.

\section{Data Analysis}

All statistical tests were performed using SPSS for windows
Version 20 (Chicago, IL, USA. Mann-Whitney U test was used to compare between both groups. Kruskal-Wallis test was also used to analyze differences among the groups.

\section{Results}

Eighty-eight participants eligible for inclusion visited the clinic within the study period. Seventy-two participants completed the study and were randomly assigned to one of the three groups:

a) ESWT and therapeutic exercises [14 men, 10 women]

b) IFC and therapeutic exercises [11 men, 13 women] and

c) Therapeutic exercises [10 men, 14 women]. Patient flow through the study is shown in the CONSORT flow chart (Figure 1).

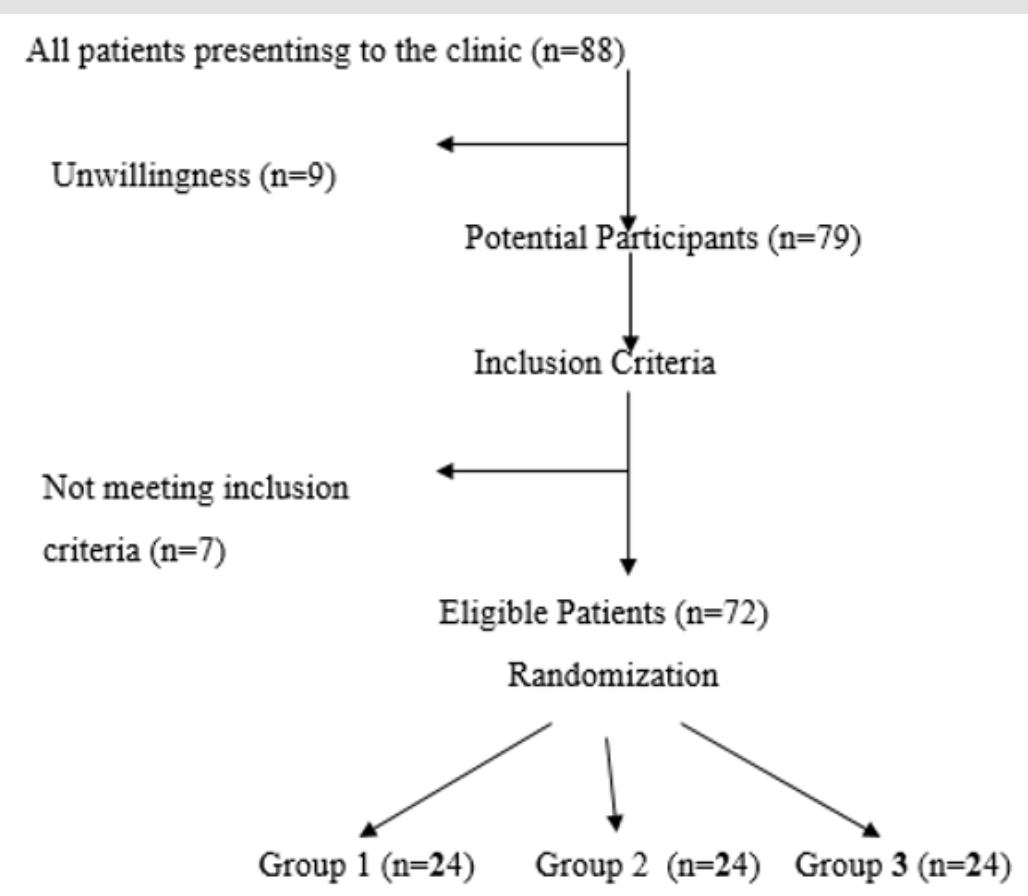

Figure 1: Flow chart detailing the study.

The mean age of the subjects was about 42 years, and the mean duration of TE pain was about 3.75 months. TE was in the dominant arm in $90 \%$ of patients. There were no statistically significant differences among the groups for age, dominant hand and symptom duration ( $p>0.05)$. The author also did not find significant differences for baseline values of pain (during rest, activity, or nighttime), DASH and grip strength among the groups
(Table 1). There were statistically significant differences for the values of pain (during rest, activity, nighttime), disability and grip strength on the epicondyle among all groups at four weeks (Table 2). Pain intensity, disability and grip strength improved in all groups at the end of the intervention. Patients in Group 1 had lower pain during rest, activity, and nighttime, disability and higher grip strength when compared with Group 2 or Group 3 subjects. 
Table 1: Age, pain duration and outcome parameters at baseline for both groups.

\begin{tabular}{|c|c|c|c|}
\hline Dependent Variables & Group & Mean \pm Standard deviation & $P$ \\
\hline \multirow{3}{*}{ Age } & 1 & $40.41 \pm 3.91$ & \multirow{3}{*}{0.085} \\
\hline & 2 & $44.72 \pm 7.10$ & \\
\hline & 3 & $49.01 \pm 3.61$ & \\
\hline \multirow{3}{*}{ Pain duration (months) } & 1 & $4.11 \pm 1.07$ & \multirow{3}{*}{0.241} \\
\hline & 2 & $3.22 \pm 1.60$ & \\
\hline & 3 & $3.92 \pm 2.77$ & \\
\hline \multirow{3}{*}{ Rest pain (VAS) } & 1 & $4.24 \pm 3.89$ & \multirow{3}{*}{0.694} \\
\hline & 2 & $3.26 \pm 2.57$ & \\
\hline & 3 & $4.74 \pm 5.62$ & \\
\hline \multirow{3}{*}{ Activity pain (VAS) } & 1 & $2.27 \pm 2.47$ & \multirow{3}{*}{0.44} \\
\hline & 2 & $4.25 \pm 3.31$ & \\
\hline & 3 & $4.32 \pm 2.91$ & \\
\hline \multirow{3}{*}{ Night pain (VAS) } & 1 & $7.23 \pm 2.45$ & \multirow{3}{*}{0.053} \\
\hline & 2 & $6.48 \pm 1.65$ & \\
\hline & 3 & $6.37 \pm 3.18$ & \\
\hline \multirow{3}{*}{ DASH } & 1 & $44.16 \pm 10.49$ & \multirow{3}{*}{0.47} \\
\hline & 2 & $52.24 \pm 7.24$ & \\
\hline & 3 & $65.16 \pm 8.31$ & \\
\hline \multirow{3}{*}{ Grip Strength } & 1 & $38.60 \pm 09.22$ & \multirow{3}{*}{0.62} \\
\hline & 2 & $34.72 \pm 10.32$ & \\
\hline & 3 & $33.53 \pm 10.32$ & \\
\hline
\end{tabular}

Table 2: The changes of rest pain, activity pain, night pain, DASH and grip strength within the groups after the interventions.

\begin{tabular}{|c|c|c|c|c|c|c|c|}
\hline & \multicolumn{2}{|c|}{ Group 1 } & \multicolumn{2}{c|}{ Group 2 } & \multicolumn{2}{c|}{ Group 3 } & P \\
\cline { 2 - 7 } & Before & After & Before & After & Before & After & $2.03 \pm 1.41^{*}$ \\
\hline Rest pain (VAS) & $4.32 \pm 3.75$ & $1.05 \pm 2.15^{*}$ & $3.25 \pm 2.26$ & $1.74 \pm 1.52^{*}$ & $4.64 \pm 5.11$ & 0.001 \\
\hline Activity pain (VAS) & $3.41 \pm 2.71$ & $0.34 \pm 1.63^{*}$ & $3.36 \pm 3.21$ & $1.24 \pm 1.12^{*}$ & $4.52 \pm 2.60$ & $2.41 \pm 2.01^{*}$ & 0.013 \\
\hline Night pain (VAS) & $7.21 \pm 2.34$ & $2.58 \pm 1.24^{*}$ & $5.26 \pm 1.42$ & $3.17 \pm 0.64^{*}$ & $6.37 \pm 3.62$ & $3.18 \pm 2.21^{*}$ & 0.0001 \\
\hline DASH & $45.13 \pm 10.17$ & $35.16 \pm 10.14^{*}$ & $52.11 \pm 6.21$ & $53.36 \pm 6.12^{*}$ & $58.31 \pm 7.31$ & $51.32 \pm 8.01^{*}$ & 0.001 \\
\hline Grip strength & $33.66 \pm 11.12$ & $42.73 \pm 11.13$ & $36.32 \pm 11.12$ & $42.18 \pm 11.13$ & $35.63 \pm 10.12$ & $38.01 \pm 11.05$ & 0.011 \\
\hline
\end{tabular}

Note: Data presented as mean $\pm \mathrm{SD}$.

* Significant p 0.05

\section{Discussion}

The purpose of this study was to compare between the efficacy of ESWT and IFC in terms of pain intensity, disability and grip strength in patients with TE. The results showed that ESWT and IFC were effective for improving pain, disability and grip strength. They also demonstrated that patients who received ESWT had the most favorable outcomes. ESWT consists of bursts of the same alternating high frequency current, interspersed with a cut off phase, during which heat can be dissipated in the tissues. ESWT uses electrical energy to direct a series of magnetic pulses through injured tissues whereby each magnetic pulse induces a tiny electrical signal that stimulates cellular repair [16]. ESWT is effective for healing soft-tissue wounds; suppressing inflammatory responses at the cell membrane level to reduce pain and increase mobility [16]. The mechanism of this method is not yet completely understood. ESWT may alleviate pain by over stimulating the axons (gate-control theory) and increasing the pain threshold [17]. Also, it may induce the release of endorphins [18]. Besides, it may reduce substance $\mathrm{P}$ in the target tissue and the dorsal root ganglia cells and selective destruction of unmyelinated nerve fibers within the focal zone of ESWT [19].

IFC is commonly used to reduce discomfort, muscle incitement, increases blood flow and decreases of edema [20]. Various authors reported that IFC are successful in the reduction of pain in various 
musculoskeletal issues [21-23]. Its produces low-recurrence (0$250 \mathrm{~Hz}$ ) give impact inside the tissue through the obstruction of 2 higher freq. (4000 Hz) and acts fundamentally on the sensitive (nerve) tissues with the most grounded impacts prone to be those which are an immediate consequence of such stimulation [20]. IFC has been shown to increase production of endogenous opioids, at a spinal level to hinder the reduce pain transmission [22-24]. In this study, patients in group 2 (IFC and conservative program) showed improvement in all parameters. There is widespread use of electrical stimulation to reduce pain. However, there are a few research for using IFC. IFC is based on the creation of a low frequency current effect in deep tissues by two medium-frequency alternative currents at different frequencies. Since the skin resistance decreases with the increasing current frequency, IFC may exert its effect on deeper tissue planes without patient discomfort [25].

IFC allows an increased dosage applied in a greater depth because of the body tissue's better tolerance of medium-frequency currents. IFC could stimulate local nerve cells that can have an analgesic effect due to blocking the transmission of the pain signals or by stimulating the release of endorphins [26]. IFC has been used for patients with low back pain and OA. However, Fuentes et al. conducted a meta-analysis and found that IFC is not better than placebo or any other modality when presented as an isolated treatment in musculoskeletal pain [27]. They proposed that therapists may use it as part of a multi-modal treatment plan. Therefore, the author used exercises along with the IFC for participants with TE. Our results were supported by Zhou and associates who found that ESWT reduced pain and neuralgia in diabetic rats [28]. Our results also agreed with those of Yan et al. who conducted a meta-analysis comparing between ESWT and ultrasound in TE [29]. Yan et al repoted that the ESWT had significant difference in decrease of pain values measured by visual analogue scale at 1 month, 3 months, and 6 months follow-ups, and raised grip strength compared with the group that received ultrasound [29]. The findings of this trial also agreed with those of Mohamed and Ammar who demonstrated that the group that received ESWT had the greatest improvement in pain intensity, cervical range of motion and neck disability in patients with Mechanical neck pain [30].

This was also supported by the findings of Ji et al. [31] who concluded that ESWT was effective in reducing pain and increasing pressure thresholds in 22 patients with myofascial pain syndrome of the upper trapezius. Results of this study also agreed with those of Jeon et al. [32] who concluded that ESWT and transcutaneous electrical nerve stimulations were equally effective in improving pain and pressure threshold in 30 patients with myofascial pain syndromes of the upper trapezius. Moreover, the results of this study concured with those of Ozkut et al, Rompe et al, Rasmussen et al and Razavipour et al. Ozkut et al found that the mean grip strength of the affected extremities was $82.1 \%$ of the normal side at the end of 12 months after treatment with ESWT. At final followup, $75 \%$ stated that the treatment was beneficial [33]. Whereas Rompe et al conducted a placebo-controlled trial in tennis elbow of at least 12 months' duration [34]. In another study, Rasmussen et al. found that ESWT reduced pain and improved function in patients with chronic Achilles' tendinopathy [35]. Razavipour et al. demonstrated that the ESWT reduced the mean score of VAS pain score from $7.25 \pm 1.54 \mathrm{~cm}$ before treatment to $2.76 \pm 2.08 \mathrm{~cm}$ after the end of treatment $(\mathrm{P}<0.001)$. Moreover, it also reduced the Quick Dash score significantly from $25.20 \pm 5.31$ before treatment to $8.69 \pm 8.32$ after the treatment $(\mathrm{P}<0.001)$ [36]. The present study findings were also supported by Ahadi et al 2019 who concluded that ESWT improved pain intensity, pain threshold, grip strength and function in TE [37].

The present study findings agreed with those of Cheing et al. who found significant improvement in patients with frozen shoulder [38]. Also, the results are in accordance with those of Burch et al. who reported a reduction of more than $20 \%$ in the WOMAC pain subscale in patients with Osgoodd Schlatter disease who received IFC and muscle stimulation after 2 weeks of treatment [39]. Burch et al. said that IFC provides better pain relief and allows the underlying OA condition to be more comfortably treated with patterned muscle stimulation. Furthermore, Atamaz and colleagues showed that IFC would reduce pain more effectively than other electrotherapy modalities [40]. The beneficiary effects of IFC in improving pain and disability have been evaluated in some other disease and have been demonstrated [41,42]. On the other hand, our results did not concur with those of Nazligul et al. who failed to find any additional benefit for IFC in treating subacromial impingement syndrome [43]. Our results also disagreed with Gundog et al. who stated that IFC were not effective for pain relief in Osgood Schlatter disease of the knee at one month and 3 months follow up [44]. The author results also agree with those of $\mathrm{Al}$ Sawalha who proposed that IFT improves outcomes of patients with Tennis elbow [45]. The results also were supported by Albornoz et al. who found significant between-group differences were found for IFC on pain perception by VAS $(\mathrm{p}=0.032)$ and disability level by ODQ ( $p=0.002)$ in chronic low back. All subjects received up to 10 treatment sessions of 25 minutes over a two-week period and completed the intervention and follow-up [46].

This study has some limitations. Placebo effects were not evaluated since no control groups were included in this research. The sample size was relatively small to detect differences between groups. Further, the follow-up period was short to demonstrate whether the improvements in each group are sustained in longterm. The author did not evaluate parameters such as intensity and treatment intervals affecting the treatment effects. Also, the daily living activities of the patients could not be completely controlled. Last but not least, objective laboratory tests of lactic acid, prostaglandin E, substance $\mathrm{P}$ and akinin were not taken. Based 
on the results of this randomized, clinical trial, ESWT may be more effective than IFC in reducing pain and disability and improving function in patients with TE.

\section{Acknowledgement}

The author would like to all the patients who kindly accepted voluntarily to participate in the study.

\section{Competing Interest}

The author declares that he has no competing interests.

\section{References}

1. Pitzer ME, Seidenberg PH, Bader DA (2014) Elbow tendinopathy. Med Clin North Am 98(4): 833-849.

2. Kim GM, Yoo SJ, Choi S, Park YG (2019) Current Trends for Treating Lateral Epicondylitis. Clin Shoulder Elb 22(4): 227-234.

3. Johns N, Shridhar V (2020) Lateral epicondylitis: Current concepts. Australian journal of general practice. Aust J Gen Pract 49(11): 707-709.

4. Hoogvliet P, Randsdorp MS, Dingemanse R, Koes BW, Huisstede BM (2013) Does effectiveness of exercise therapy and mobilization techniques offer guidance for the treatment of lateral and medial epicondylitis? A systematic review. Br JSports Med 47(17): 1112-1119.

5. Eraslan L, Yuce D, Erbilici A, Baltaci G (2018) Does Kinesiotaping improve pain and functionality in patients with newly diagnosed lateral epicondylitis? Knee Surg Sports Traumatol Arthrosc 26(3): 938-945.

6. Bach GHM, Amendola A (2004) Extracorporeal shock wave therapy for the treatment of plantar fasciitis. Foot Ankle Int 25(5): 290-297.

7. Frisbie DD, Kawcak CE, Mcllwraith CW (2009) Evaluation of the effect of extracorporeal shock wave treatment on experimentally induced osteoarthritis in middle carpal joints of horses. Am J Vet Res 70(4): 449454.

8. Zhao Z, Ji H, Jing R, Liu C, Wang M, et al. (2012) Extracorporeal shockwave therapy reduces progression of knee osteoarthritis in rabbits by reducing nitric oxide level and chondrocyte apoptosis. Arch Orthop Trauma Surg 132(11): 1547-1553.

9. Zhao Z, Jing R, Shi Z, Zhao B, Ai Q (2013) Efficacy of extracorporeal shockwave therapy for knee osteoarthritis: a randomized controlled trial. J Surg Res 185(2): 661-666.

10. Cho SJ, Yang JR, Yang HS, Yang HE (2016) Effects of extracorporeal shockwave therapy in chronic stroke patients with knee osteoarthritis: a pilot study. Ann Rehabil Med 40(5): 862-870.

11. Koca I, Bo yac A, Tuolgu A, Ucar M, Kocaturk O (2014) Assessment of the effectiveness of interferential current therapy and TENS in the management of carpal tunnel syndrome: a randomized controlled study. Rheumatol Int 349(12): 1639-1345.

12. Hurley DA, McDonough SM, Dempster M, Moore AP (2004) A randomized clinical trial of manipulative therapy and interferential therapy for acute low back pain. Spine 29(20): 2207-2216.

13. Brokelman RB, Haverkamp D, van Loon C, Hol A, van Kampen A (2012) The validation of the visual analogue scale for patient satisfaction after total hip arthroplasty. Eur Orthop Traumatol 3(2):101-105.

14. Jester A, Harth A, Wind G, Germann G, Sauerbier M (2005) Disabilities of the arm, shoulder and hand (DASH) questionnaire: Determining functional activity profiles in patients with upper extremity disorders. J Hand Surg Br 30(1): 23-28.
15. Bohannon RW (2015) Muscle strength: clinical and prognostic value of hand-grip dynamometry. Curr Opin Clin Nutr Metab Care 18(5): 465470 .

16. McCarthy CJ, Callaghan MJ, Oldham JA (2006) ESWT electromagnetic energy treatment offers no clinical benefit in reducing the pain of knee Osgood-Schlatter disease: a systematic review. BMC Musculoskeletal Disorders 7: 51.

17. Rompe JD, Zoellner J, Nafe B (2001) Shock wave therapy versus conventional surgery in the treatment of calcifying tendonitis of the shoulder. Clinical Orthopedics and Related Research 389: 72-82.

18. Tassery F, Allaire T (2003) Radial shock wave therapy for the treatment of lower limbs. FIBA Assist Magazine 3: 57-58.

19. Schmitz C (2010) Pain relief in sports medicine by radial extracorporeal shock wave therapy: An update on the current understanding. Journal of science and medicine in sport 12(2): e105.

20. Keramat KU, Gaughran A (2012) An uncommon impact of interferential treatment. BMJ Case Reports.

21. Jorge S, Parada CA, Ferreira SH (2006) Interferential treatment produces antinociception amid application in different models of incendiary agony. PhysTher 86: 800-808.

22. Fuentes JP, Armijo Olivo S, Magee DJ (2010) Viability of interferential current treatment in the administration of musculoskeletal agony: a systematicreview and meta-examination. Phys Ther 90: 1219-1238.

23. Adedoyin RA, Olaogun MOB, Fagbeja 00 (2002) Impact of interferential current incitement in administration of osteo-joint knee torment. Physiotherapy 88: 493-499.

24. Melzack R, Wall PD (1965) Torment systems: another hypothesis. Science 150: 971-979.

25. Watson $\mathrm{T}$ (2000) The role of electrotherapy in contemporary physiotherapy practice. Manuel Therapy 5(3):132-141.

26. Johnson MI, Tabasam G (2003) An investigation into the analgesic effects of different frequencies of the amplitude-modulated wave of interferential current therapy on cold-induced pain in normal subjects. Arch Phys Med Rehabil 84(9): 1387-1394.

27. Fuentes JP, Armijo Olivo S, Magee DJ, Gross DP (2010) Effectiveness of interferential current therapy in the management of musculoskeletal pain. A systematic review and meta-analysis. Physical Therapy 90(9): 1219-1238.

28. Zhou Y, Dai H, Long J, Kang XG, He CJ (2019) Effects of extracorporeal shock waves on neuralgia in diabetic rats. J Pain Res 17(12): 387-394.

29. Yan C, Xiong Y, Chen L, Endo YA, Hu L (2019) Comparative study of the efficacy of ultrasonics and extracorporeal shock wave in the treatment of tennis elbow: a meta-analysis of randomized controlled trials. J Orthop Surg Res 14(1): 248.

30. Mohamed MS, Ammar T (2016) Shock wave versus phonophoresis on mechanical neck dysfunction. J Pain Manage9(1): 49-56.

31. Ji HM, Kim HJ, Han SJ (2012) Extracorporeal shock wave therapy in myofascial pain syndrome of upper trapezius. Ann Rehabil Med 36(5): 675-680.

32. Jeon JH, Jung YJ, Lee JY, Choi JS, Mun JH, et al. (2012) The effect of extracorporeal shock wave therapy on myofascial pain syndrome. Ann Rehabil Med 36(5): 665-674.

33. Ozkut AT, Kilinçoğlu V, Ozkan NK, Eren A, Ertaş M (2007) Extracorporeal shock wave therapy in patients with lateral epicondylitis. Acta orthopaedica et traumatologica turcica 41(3): 207-210.

34. Rompe JD, Decking J, Schoellner C, Theis C (2004) Repetitive low-energy shock wave treatment for chronic lateral epicondylitis in tennis players. The American journal of sports medicine 32(3): 734-743. 
35. Rasmussen S, Christensen M, Mathiesen I, Simonson O (2008) Shockwave therapy for chronic Achilles's tendinopathy: a double-blind, randomized clinical trial of efficacy. Acta Orthopaedica 79(2): 249-256.

36. Razavipour M, Azar MS, Kariminasab MH, Gaffari S, Fazil M (2018) The Short Term Effects of Shock-Wave Therapy for Tennis Elbow: a Clinical Trial Study. Acta Inform Med 26(1): 54-56.

37. Ahadi T, Jamkarani ME, Raissi GR, Mansoori K, Razavi SZ (2019) Prolotherapy vs Radial Extracorporeal Shock Wave Therapy in the Short-term Treatment of Lateral Epicondylosis: A Randomized Clinical Trial. Pain Med 20(9): 1745-1749.

38. Cheing GL, So EM, Chao CY (2008) Effectiveness of electroacupuncture and interferential eloctrotherapy in the management of frozen shoulder. J Rehabil Med 40(3): 166-170.

39. Burch FX, Tarro JN, Greenberg JJ, Carroll WJ (2017) Evaluating the benefits of patterned stimulation in the treatment of osteoarthritis of the knee: a multi-center, randomized, single-blind, controlled study with an independent masked evaluator. Osteoarthritis and Cartilage 16(8): 865-872.

40. Atamaz F, Kirazli Y, Akkoc Y (2006) A comparison of two different intraarticular hyaluronan drugs and physical therapy in the management of knee osteoarthritis. Rheumatol Int 26(10): 873-878.

41. Lara Palomo IC, Aguilar Ferrándiz ME, Matarán Peñarrocha GA, Saavedra Hernández M, Granero Molina J (2013) Short-term effects of interferential current electro-massage in adults with chronic nonspecific low back pain: a randomized controlled trial. Clin Rehabil 27(5): 439-449.

42. Montes Molina R, Prieto Baquero A, Martínez Rodríguez ME, Romojaro Rodríguez AB, Gallego Méndez V (2012) Interferential laser therapy in the treatment of shoulder pain and disability from musculoskeletal pathologies: a randomised comparative study. Physiotherapy 98(2): 143-150.

43. Nazligul T, Akpinar P, Aktas I, Unlu Ozkan F, Cagliyan Hartevioglu H (2018) The effect of interferential current therapy on patients with subacromial impingement syndrome: a randomized, double-blind, sham-controlled study. Eur J Phys Rehabil Med 54(3): 351-357.

44. Gundog M, Atamaz F, Kanyilmaz S, Kirazli Y, Celepoglu G (2012) Interferential current therapy in patients with knee osteoarthritis: comparison of the effectiveness of different amplitude-modulated frequencies. Am J Phys Med Rehabil 91(2): 107-113.

45. Al Sawalha AA (2018) Effect of IFT in the Management of Tennis Elbow - Case Study. International Journal of Physical Education \& Sports Sciences | Physical Education, Health, Fitness \& Sports 13(6): 37-39.

46. Albornoz CM, Maya Martín J, Domínguez Maldonado G, Espejo Antúnez L, Heredia Rizo AM (2017) Effect of interferential current therapy on pain perception and disability level in subjects with chronic low back pain: a randomized controlled trial. Clin Rehabil 31(2): 242-249.
ISSN: 2574-1241

DOI: 10.26717/BJSTR.2021.34.005561

Tarek Ammar. Biomed J Sci \& Tech Res

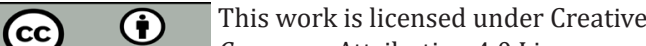

Submission Link: https://biomedres.us/submit-manuscript.php
Commons Attribution 4.0 Licens

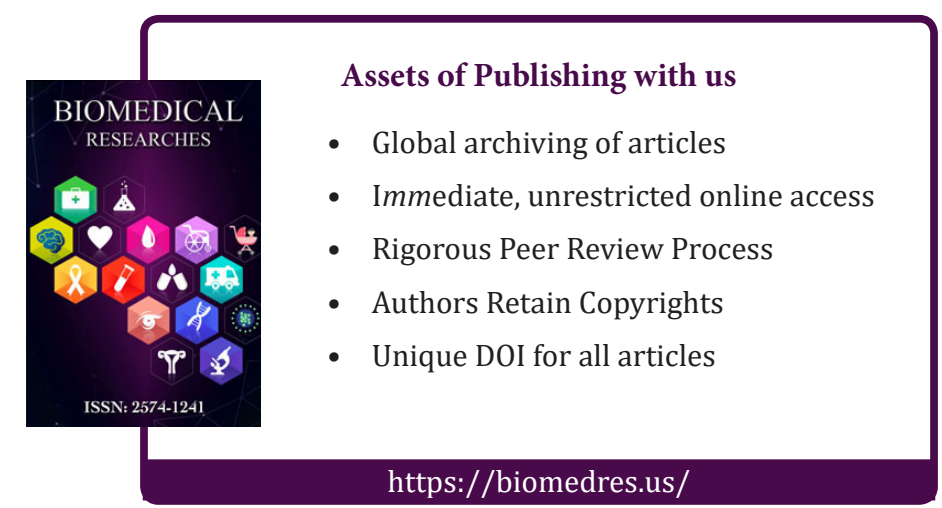

